\title{
Author index to Volume 84
}

\section{Key to abbreviations:}

(BR) Book reviews; (L) Letters to the editors; (Suppl 1) Supplement 1; (Suppl 2) Supplement 2

Aaltonen LA 1314

Abbruzzese A 1586

Abe N 1317

Abe T 824

Ablett S 11

Achard JL 31 (Suppl 2)

Achtelik W 1036

Adenis A 65 (Suppl 2)

Adolfsson J 329, 1505

Adrian TE 926

Agus V 1087

Ahn BW 844

Aiken R 157

Aird R 600

Akerley W 157

Akeshima R 1551

Akhmedkhanov A 975

Akiba H 920

Akre K 965

Ala-aho R 659

Albers P 1330

Albery IP 1172

Alcock HE 499

Alfaro M 1219

Allemann J 33

Allen J 157

Allinen M 116

Allredl DC 493

Alsner J 1070

Altermatt HJ 1372

Alvarnas J 1591

Amos CI 1182

Andersen A 397

Andersson SW 1193

Andre F 903

Andreoli F 878

Andrulis IL 1635

Angus B 270

Aoki M 1610

Appierto V 1528

Arab MO 226

Arakawa S 859

Arbuck S 1424

Ardavanis A 1488

Arditti J 604

Argenio GF 636

Armstrong A 1433

Arnogiannaki N 1488

Arnold JM 352

Aromaa A 122

Arora A 340

Arseneau J 17 (Suppl 1)

Asami H 1242

Aschele C 1023

Askling J 113

Asselain B 24 (Suppl 2)

Assouline D 1179

Atkinson R 170

Atomi Y 1317
Aubele M 202

Austrup F 1664

Autillo-Touati A 360

Azzarelli A 1087

Baccino FM 946

Bachelot T 18 (Suppl 2)

Baelde JJ 535

Bah E 1207

Bahl A 1143

Baidas S 157

Bailly C 31 (Suppl 2),

81 (Suppl 2)

Baldelli AM 470

Balducci A 722

Ballinger JR 367

Balmaceda I 1219

Banerjee S 975

Bar Meir S 475

Baria K 892

Barletta E 38

Barni S 470

Baron JA 714

Bartels T 813

Bartholomew C 1630

Barzelloni ML 38

Bassi C 253

Bassilopoulos P 1488

Basuyau JP 18 (Suppl 2), 24

(Suppl 2), 31 (Suppl 2)

Bataillard A 8 (Suppl 2), 24

(Suppl 2), 31 (Suppl 2)

Batchelor T 157

Batlle G 1215

Batsis C 1602

Bauchinger M 202

Baudin E 808

Bayer P 344

Bayoumy S 1182

Bearzatto A 1387

Beckendorf V 81 (Suppl 2)

Beckman RA 1043

Begent RHJ 1671

Beijnen JH 42

Belizário JE 1135

Bell CMJ 435

Bell RS 1635

Beloueche M 691

Belyakov OV 674

Ben Ayed F 783

Bender DP 1624

Bengtsson C 1193

Benhamiche AM 1477

Benharkat S 783

Benicke M 982

Bennouna J 1179

Bensadoun RJ 42 (Suppl 2)

Bentzen SM 1084

Bergenbrant S 621
Berger J 819

Bergeron C 360

Berggren P 1505

Berghmans T 1150

Berney DM 340

Bernhard J 1156

Bernini GP 636

Bertani H 881

Berthois Y 936

Bertler SZ 157

Bessell EM 1293

Best N 1482

Betticher DC 1372

Bevan S 1314

Bey P 55 (Suppl 2)

Bezold G 72

Bharaj B 760

Bhargava P 1599

Bianchi P 196

Bibby MC 290

Bible KC 1391

Bicknell D 1314

Bicknell R 558

Bidart JM 808

Bijker N 539

Bijman MNA 1115

Bilancia D 38

Birch JM 141

Birembaut P 1616

Bissett D 308

Björkholm M 621

Blackhall FH 465

Blanc-Vincent, MP 8 (Suppl 2), 37 (Suppl 2), 42 (Suppl 2)

Blay JY 78 (Suppl 2)

Blizzard L 392

Blomqvist C 704, 1047

Blomqvist CP 244

Boccia R 17 (Suppl 1)

Boćko D 1339

Boddy AV 11, 452, 1029

Bode U 1453

Bodmer WF 1314

Boehm DA 571

Boerner SA 1391

Boffi B 878

Boggi U 1535

Boher J-M 903

Boige V 631

Bokemeyer C 313

Bond JA 520

Bondy ML 1182

Bonnier P 783

Borner MM 1372

Borrello S 529

Bory J-P 1616

Bosch CAJ 539

Boshoff C 1008 (BR)

Boussin FD 631
Boven E 550

Bowman A 600

Bowman KJ 106

Boyle P 1472

Brada M 148 (BR)

Braithwaite KL 270

Bramald S 1002

Brampton M 11

Branle F 1150

Bratti MC 1219

Brémond A 24 (Suppl 2),

31 (Suppl 2)

Brindle KM 832

Brinkmann K 697

British National Lymphoma

Investigation and Central

Lymphoma Group 1293

Brosman S 24 (Suppl 1)

Browman G 1 (Suppl 2)

Brown JM 19

Brown NJ 1384

Browne JK 3 (Suppl 1)

Brugère J 37 (Suppl 2)

Brunat-Mentigny M 78

(Suppl 2)

Brunetti C 1166

Bubeník J 374

Bucana CD 844

Buckley TJD 1443

Buisson M 783

Bukowski R 24 (Suppl 1)

Bull JH 1512

Bull S 1635

Bullerdick J 381

Bunch K 1460

Burk RD 1219

Burniat A 1150

Burton A 1293

Buttafoco P 881

Buyse IM 743

Cabal-Manzano R 1599

Cacciatore B 897

Caldas C 193

Calogero A 1115

Calvert AH 106, 452

Calvert H 1443

Cameron DA 1146

Campbell NC 910

Campbell T 157

Cancer Research Campaign

Phase I/II Committee 465

Canevari S 1528

Cano J-P 1677

Capelli P 253

Capkun V 1227

Caputo S 1535

Caraglia M 1586

Carder PJ 1095 
Carmichael J 1443

Caroti C 1023

Carreca I 1166

Carella AM 55 (Suppl 2)

Carrie C 78 (Suppl 2)

Cartwright RA 141

Casaretti R 470

Casciari JJ 1544

Cascinu S 470

Cassidy J 308, 910

Castaigne D 24 (Suppl 2)

Castle PE 1219

Catalano G 470

Catalano V 470

Catalin J 604

Caux V 482

Cavadini E 1528

Cavenagh JD 147 (L)

Cemazar M 565

Chamberlain MC 157

Chan D 24 (Suppl 1)

Chan FKL 335

Chaplin DJ 565, 626

Chastagner P 604

Chatelain P 360

Chatenoud L 722

Chauvel P 42 (Suppl 2)

Chauvergne J 24 (Suppl 2)

Chauvot P 78 (Suppl 2)

Chay KO 844

Chen H-L 1556

Chen J 1265

Chen Q 1520

Chen WY 441 (L)

Chen-Shtoyerman R 475

Chenevix-Trench G 352

Cheng KK 1215

Cherny N 587

Chilvers CED 413

Chin S-F 193

Chiu S-M 1099

Cho DY 186

Cho M-S 1252

Cho YK 186

Choi J-H 186

Choong PFM 951

Chow W 1591

Christen RD 1571

Christensen M 1084

Christian M 1424

Chuang LY 709

Chung SCS 335

Ciccarelli A 1586

Ciccolini J 1677

Cini G 878

Cioffi R 38

Cirillo M 1023

Ciszak L 1339

Clarke K 1322

Clarke NW 1417

Clavel C 1616

Clendeninn N 11

Cleris L 1528

Cleton-Jansen AM 535

Cluck MW 926

Coates AS 1156

Coebergh JWW 700

Cohen-Solal C 18 (Suppl 2)

Cohn A 157

Colangelo D 1387
Colella G 1387

Coleman R 170

Coleman RE 1126

Collard TJ 520

Collier M 308

Collingridge DR 626

Collins A 429

Collins HM 1664

Colowick AB 17 (Suppl 1), 24 (Suppl 1)

Colton T 126

Comella G 470, 1166

Comella P 1166

Commo F 504

Comoy E 808

Conroy T 65 (Suppl 2)

Corbo M 1397

Corcoran JJ 489

Cornbleet MC 1146

Corpet DE 90

Cortesi E 1023

Coshic O 1016

Cosset JM 55 (Suppl 2)

Costa A 651

Costelli P 946

Cotter FE 1397

Cowan R 8

Craft JA 1630

Cramer DW 714

Cranston D 558

Crawford M 170

Creaney J 52

Crépin M 802

Croucher PI 1126

Cruickshank M 170

Cull A 594

Cullen MH 1293

Cummings J 600

Cummins C 1215

Cunningham D 1

Cupissol D 81 (Suppl 2)

Cuq P 1677

Curtin NJ 106

Cutuli B 55 (Suppl 2)

Dabit D 504

Dachez R 360

Dai Q 994

Daidone MG 651, 1387

Dalley CD 147 (L)

Dame MK 1076

Dancourt V 1477

Danesi R 1535

d'Anjou J 31 (Suppl 2)

Darby S 728

Dassonville O 42 (Suppl 2)

Dauplat J 18 (Suppl 2)

Davies MPA 1064

Day N 423

de Conno F 587

de Haes JCJM 1577

De Jaeger K 1280

de Jong S 1348

de Jonge MWA 1115

de Klerk N 52

de Leij L 1115

de Leij LFMH 1348

De Lena M 651

De Paoli P 122

De Ridder M 1122
De Tullio R 946

de Vries EGE 1348

Debuiche S 8 (Suppl 2)

DeCataldis G 1166

Decker S 1424

Deguchi H 915

Del Tacca M 1535

Delahaye L 1115

Delattre O 199

Dellapasqua S 651

Demard F 37 (Suppl 2), 42 (Suppl 2)

Demetri G 17 (Suppl 1)

Demetri GD 31 (Suppl 1)

Depierre A 903

D'Erasmo E 636

Dermime S 1433

Descamps P 31 (Suppl 2)

DeVries A 179

Dey P 8

Di Paolo A 1535

Di Pietro A 1405

Diamandis EP 643, 760

Dick GS 1029

Diehl V 381

Diel IJ 344

Dillner J 122

Diociaiuti L 460

Dodd HE 141

Doll R 728

Dombernowsky P 1301

Dong C 388

Doré JF 81 (Suppl 2)

Doroshow JH 1591

Dorval T 81 (Suppl 2)

dos-Santos-Silva I 406

Douillard JY 1179

Doz F 604

Drummond F 218

Dubois JB 24 (Suppl 2)

Dubreuil A 579

Duchateau L 539

Duffy S 423

Dugani A 881

Dummer R 1036

Durand-Zaleski I 4

(Suppl 2)

Durrant LG 80, 1443

Duval C 539

Duvillard P 18 (Suppl 2)

Dwivedi SN 1016

Dwyer T 392

Eardley A 8

Earle CC 441 (L)

East Anglian Breast Screening

Programme 423

Easton D 388

Edwards YH 218

Eerola H 116, 704

Eggleton P 170

Egrie JC 3 (Suppl 1)

Ehrhardt A 813

Eisenbrand G 283

Eissa S 1182

Ekbom A 113

Ekström AM 965

El-Abbadi M 1107

El-Badawy SA 1182

Elgie AW 680
El-Hariry I 1656

Elias D 74 (Suppl 2)

Elliott AM 910

Elliott P 1482

Ellis LM 844

Ellison G 1512

Ellwanger U 1036

Elomaa I 1047

Eng C 748

Engert D 202

Esteve J 1477

Estlin EJ 11, 1029

Etienne M-C 579

European Lung Cancer Working

Party (ELCWP) 1150

Evans HS 435

Evrard A 1677

Exton MS 1265

Fait E 1354

Faivre J 1477

Faivre S 631

Falconi M 253

Falk S 1143

Fallowfield L 48, 1011

Fang W 743

Farsi F 8 (Suppl 2),

31 (Suppl 2), 78 (Suppl 2)

Faucher A 37 (Suppl 2)

Fels LM 313

Fennell DA 1397

Fenwick J 452

Ferdeghini M 636

Feretti I 881

Ferme C 55 (Suppl 2)

Fernandez E 722

Fervers B 8 (Suppl 2),

18 (Suppl 2), 24 (Suppl 2),

31 (Suppl 2), 51 (Suppl 2),

55 (Suppl 2), 78 (Suppl 2),

81 (Suppl 2)

Fey M 1036

Fidder HH 475

Fielding K 1443

Figer A 475,478

Fina $\mathrm{F} 783$

Fischel J-L 579

Fisher JL 951

Flavell DJ 571

Flavell SU 571

Fleischhack G 1453

Fleishman A 24 (Suppl 1)

Fleming D 170

Flex D 478

Foekens JA 783

Folkard M 674

Fonatsch C 381

Fonderico F 1586

Fondrinier E 24 (Suppl 2), 31 (Suppl 2)

Foray N 1272

Forman SJ 1591

Formelli F 1528

Formento P 579

Fouret P 504

Fracchioli S 643

Franceschi S 722

Francesconi D 878

Francis RJ 1671

François E 61 (Suppl 2) 
Frappaz D 604

Frasci G 1166

Frassineti GL 1023

Friedel A 283

Friedman E 475, 478

Frontini L 38, 470

Fry A 594

Frydecka I 1339

Fuchimoto S 443

Fueyo J 1252

Fuggle S 558

Fujita H 836

Fujita T 1564

Fujita Y 824, 836

Fukiyama C 558

Fukuda R 1640

Fulton D 157

Fulton R 1630

\section{Gabriel R 1616}

Gainet M 903

Galeotti T 529

Gallateau-Sallé F 49 (Suppl 2)

Gallick GE 844

Gallo C 38

Gallo L 1023

Gao F 1185

Gao Y-T 994

Garbay JR 81 (Suppl 2)

Garbe C 1036

Gaumann A 1354

Gauthier LR 631

Gayko U 24 (Suppl 1)

Gebski V 297

Geick A 813

Gelleret N 87

Gennari L 196

Gentet JC 78 (Suppl 2), 604

Genzlinger A 283

George NJR 1417

Gérard H 783

Gerçel-Taylor Ç 1624

Gerhold M 1571

Gerken M 134

Geva R 478

Ghisdal L 1150

Giai M 760

Giammarile F 55 (Suppl 2), 78 (Suppl 2)

Guillo S 8 (Suppl 2)

Giannini F 881

Giannoudis A 1058

Gibbs A 8

Gilbert M 157

Gill L 1460

Giovannini M 74 (Suppl 2)

Girling DJ 1447

Giusti C 936

Glantz MJ 157

Glaspy J 1 (Suppl 1),

17 (Suppl 1), 24 (Suppl 1)

Glavina-Durdov M 1227

Gochi A 443

Goepel JR 499

Gokgoz N 1635

Goldacre MJ 1460

Gomez-Manzano C 1252

Gordini G 643

Gordon D 17 (Suppl 1)

Gore M 1043, 1146
Gory G 8 (Suppl 2)

Gory-Delabaere G 37 (Suppl 2), 42 (Suppl 2), 51 (Suppl 2)

Goto A 1317

Graadt van Roggen JF 535

Graham J 170

Granotier C 631

Grau B 1179

Grazia Caprio M 38

Grazia Del Buono M 881

Graziano F 470

Greenberg ER 126, 714

Greenberg HS 157

Greenberg M 1219

Gregory WM 1293

Grénman R 659

Greulich KM 72

Gridelli C 38

Griffin MJ 1029

Griffith H 24 (Suppl 1)

Griffith K 1460

Grigat JP 697

Griggs J 832

Grönberg H 429

Grossi F 1023

Grottola A 881

Gruber A 621

Gruenewald S 297

Grunfeld EA 1172

Grunicke H 1405

Guastalla JP 18 (Suppl 2),

24 (Suppl 2), 31 (Suppl 2)

Gugger M 1372

Guglielmi A 1023

Guignat L 808

Guillou P 1443

Gullick WJ 1322

Guo Z 791

Gurney EM 19

Gutheil JC 157

Guy M 520

Habu S 1235

Hagiwara A 824

Hagiwara T 737 (L)

Hagiwara Y 1564

Haglund C 1363

Haie-Meder C 24 (Suppl 2)

Haisma HJ 550

Hájková R 374

Hakama M 164

Halbert G 465

Hale JP 1029

Halford S 1314

Hall AJ 1207

Hallberg L 1193

Haller AM 1179

Haller U 33

Halter R 813

Hamajima N 1199

Hamam SM 321

Hamamoto S 1640

Hamilton SR 1182

Hammerlid E 149

Hammond DW 499

Hancock B 1146

Hancock BW 465, 499, 1293

Haniuda M 25

Hanks GW 587

Hanna M 587
Hansson J 329, 1505

Hansson L-E 965

Hara I 859

Hara S 859

Hardcastle JD 1443

Hardy, J 88 (Suppl 2)

Harlow BL 714

Harmon D 17 (Suppl 1)

Harris AL 558, 1322

Harrison R 8

Hartge P 126

Hartley JA 1671

Hartmann JT 313

Hasan C 1453

Hasegawa M 25

Hashimura M 209

Hatch EE 126

Hattori Y 1076

Haugen A 226

Hauschild A 1036

Hawkins NJ 232

Hawtin PG 413

Hearn S 1043

Heatherington AC 11 (Suppl 1)

Hebert JR 994

Hedley D 367

Heemann U 1265

Heiberg I 686

Heighway J 1372

Helgesen K 1219

Hellmann K 959

Helmerhorst TJM 796

Hemminki K 388, 969, 990, 1466, 1505

Hendry WF 340

Henriksson R 429

Henry-Amar M 55 (Suppl 2)

Hepburn PM 19

Herbst AL 126

Hermans J 64

Heros D 157

Herrero R 1219

Herrington CS 1058

Hesketh R 832

Hettmer S 1453

Hey K 1460

Hietanen P 897

Higashiyama S 1377

Higgins CD 406

Highley M 452

Hildesheim A 1219

Hill BT 290

Hill RP 1280

Hill SA 626

Hiraiwa N 1258

Hirakawa $\mathrm{H} 851$

Hirose K 1199

Ho MS 709

Hobson R 11

Hochhauser D 1671

Hodgson SV 435

Hoffstetter S 31 (Suppl 2)

Höfler H 202

Hofmann J 1405

Hogendoorn PCW 535

Holder AL 565

Holford TR 1472

Holli K 164

Hollingshead M 1424

Holm G 621
Holmberg E 429

Holwell SE 290

Honda T 25

Hong Y-K 1252

Hoover RN 126

Hopwood P 8

Horn E 344

Horsman MR 1070

Houba PHJ 550

Hough RE 499

Houlihan PS 1182

Houlston RS 1314

Houvenaeghel G 24 (Suppl 2)

Howell SB 157

Hsieh MY 709

Hu X 791

Huang S 743

Hulthén L 1193

Hunter JAA 1146

Hürny C 1156

Hutchinson M 1219

Hutchison CJ 512

Huuhtanen RL 244

Huusko P 116

Hynes H 17 (Suppl 1)

Iacoangeli M 529

Ikonen T 1344

Iliadis A 604

Im S-A 1252

Imai Y 737 (L), 886

Inada M 886

Inazawa J 824

Inoue H 276

Inoue M 1199

Inui K 263

Inui Y 886

Iqbal M 1064

Irmin L 478

Iselius L 429

Ishihara S 1640

Islam I 218

Islam-Ali BS 1648

Isogai S 1681

Isola J 164

Itamochi H 1551

Ito $\mathrm{N} 886$

Ito Y 1242, 1377

Itoh K 915

Iversen T 1463

Iwamoto Y 768

Iwatsuki K 920

Izawa M 1258

Jack A 1293

Jack AD 1207

Jackson JA 1544

Jackson LE 691

Jacob JH 61 (Suppl 2)

Jacot W 903

Jaeckle KA 157

Jaehde U 1453

Jagannathan NR 1016

Jagdev SP 1126

Jaiyesimi IA 24 (Suppl 1)

Jakic-Razumovic J 1227

Jansen SJT 1577

Janssens MY 1122

Jansson O 329, 1505

Jarup L 1482 
Jarvis C 1064

Jayson GC 465

Jelínek F 374

Jeng JE 709

Jenkins V 48, 1011

Jenkinson CM 413

Jensen OM 1070

Jerkeman M 303

Jeskanen L 659

Jewell RC 42

Jin F 994

Jodrell DI 600

Joensuu H 164

Johnson JI 1424

Johnson KJ 1076

Johnson PWM 19

Johnston A 11

Jolly K 1308

Jones A 558

Joo HJ 186

Jordan R 489

Jordan S 340

Jouve JL 1477

Julka PK 1016

Jung YD 844

Justice G 17 (Suppl 1)

Kagami I 545

Kainu T 116

Kallioniemi OP 1344

Kallioniemi O-P 116

Kalso E 587

Kalyandrug S 1424

Kamai T 1242

Kamazawa S 1551

Kameyama T 915

Kamidono S 859

Kamp S 1330

Kanamori M 1610

Kanamori Y 1551

Kaneko F 920

Kaneko S 400

Kannagi R 1258

Kanuma T 545

Kanz L 313

Kappas AM 1602

Kappeler A 1372

Karakostas K 1602

Karameris A 1488

Karoski WJ 1412

Kaskel P 72

Kassem HSh 321

Kasumi F 736 (L)

Kataoka A 276

Katballe N 1084

Kato H 851, 1235

Kato I 975

Katsaros D 643

Kaufman R 126

Kaufmann R 1036

Kaufmann SH 1391

Kavanagh M-C 1280

Kawakami S 25

Kawano Y 263

Kawashima K 1640

Kawata S 886

Kaye SB 170

Kazumori H 1640

Keck A-V 344

Keller J 1070
Kenani A 1272

Kerbrat P 18 (Suppl 2)

Kerkelä E 659

Kessler J 17 (Suppl 1)

Key T 728

Khan F 1512

Khoo K-S 1185

Khuder SA 1188

Kievit J 1577

Kigawa J 1551

Kikuchi S 920

Kim H 643

Kim HC 186

Kim HS 186

Kim J-S 1252

Kim KB 186

Kim MS 844

Kim MW 186

Kim MY 975

Kimura T 1610

Kinlen LJ 1002

Kinoshita Y 1640

Kisseljov F 791

Klein Kranenbarg E 64

Kliukiene J 397

Klocke R 813

Klokman WJ 700

Knekt P 122

Knezetic JA 926

Knop S 313

Knowles G 600

Kodaira N 1681

Koenig KL 975

Koga T 915

Kogut N 1591

Kohler J 11

Kohler R 78 (Suppl 2)

Kohn EC 1330

Koivisto PA 1344

Koivunen E 1363

Konerding MA 1354

Kontani K 1258

Koopman FJ 42

Kornacker M 381

Kosmaczewska A 1339

Kovařík J 374

Kraehn GM 72

Kreienbrock L 134

Kreuzer M 134

Kringlen E 1463

Kroesen BJ 1115

Kuba K 864

Kubo K 25

Kulka U 202

Kulke MH 748

Kumar M 1016

Kuroishi T 1199

Kuter D 17 (Suppl 1)

Kvarnung M 122

La Vecchia C 722

Labianca R 470, 1023

Lacava J 651

Ladner DP 33

Laffargue F 31 (Suppl 2)

Lafitte JJ 1150

LaFollette S 157

Lagergren J 120

Laghi L 196

Lagrange JL 49 (Suppl 2)
Lake RA 52

Lancashire RJ 141

Landriscina M 529

Lane EB 512

Lane SR 1043

Lang JC 237

Lang SH 1417

Langezaal SM 535

Lansac J 31 (Suppl 2)

Lansdown MRJ 1095

Lapidus L 1193

Larsen SS 686

Larsson J 926

Larsson P 329, 1505

Lartigau E 24 (Suppl 2)

Lashford L 11

Lassen P 65 (Suppl 2)

Lastella M 1535

Lau JYW 335

Laurberg S 1084

Laurila P 244

Le Chevalier T 903

Leach MO 691

Leblanc E 24 (Suppl 2)

Lee AV 493

Lee C 100

Lee KB 186

Lee SN 1252

Lee T-L 335

Leenders RGG 550

Legrand E 802

Lehmann M 49 (Suppl 2)

Lehnert G 982

Leidecker V 8

Leiter U 72

Lejeune C 1477

Lemaitre F 1150

Leminen A 1363

Lemoine NR 253, 1656

Leonard RCF 1437

Leone B 651

Leone R 1387

Leong LA 1591

Lerouge D 1179

Lesser G 157

Letizia C 636

Leung WK 335

Levin B 1182

Levitz M 975

Lewandowicz GM 680

Lewis CM 435

Lewis IJ 11

Lhommé C 18 (Suppl 2), 24 (Suppl 2)

Li F 25

Li L 80

Li M 263

Li X 969

Liaras J 360

Lim H-Y 186

Limoli CL 489

Lin X 1571

Lin ZY 709

Linch DC 1293

Linder S 329

Lindner H 1330

Linse R 1036

Lister TA 147 (L)

Little J 910

Little MA 1029
Liu F 1556

Liu R 1405

Liu T-J 1252

Liu W 844

Loda M 748

Logue JP 8

Lohi J 659

Longmate J 1591

Longo G 303

Lorenz M 1602

Lorenzato M 1616

Lorigan PC 499

Lorincz AT 1219

Lorite MJ 1135

Lotan R 1528

Louwman WJ 700

Lovas $\mathrm{S} 926$

Lu P 1405

Ludvíková V 374

Ludwig H 344

Lukas P 179

Lukish JR 1182

Lund Nilsen TI 417

Lunec J 270

Luny D 512

Lupoli G 1586

Lykkesfeldt AE 686

Maartense E 1577

McCann J 423

Macchia M 1535

McDowell H 11

McGill A 452

McGown AT 465

Machin D 1185

Mackie PS 951

Mackie RM 1146

McKinney PA 141

McLaren BM 52

McLean C 600

McLean S 512

Maclennan KA 1293

McLeod HL 308

McQuay HJ 587

Maehara N 864

Maher EJ 1172

Maislinger S 179

Mäkelä P 1047

Malcolmson AM 674

Malesci A 196

Malingré MM 42

Malmer B 429

Manenti F 881

Manfredi MG 1107

Mangeonjean C 1616

Mann JR 141

Manno M 881

Manzione L 38

Manzotti C 1387

Marchetti A 1535

Margison GP 321

Margolin KA 1591

Maria B 157

Maric R 1215

Marinov J 374

Marko D 283

Marshall J 1599

Martignetti A 1586

Martin PM 783

Martin P-M 936 
Marttunen MB 897

Maruyama Y 25

Masaki T 1317

Mascaux C 1150

Mason W 157

Massobrio M 643

Massoudi N 381

Masters JRW 1686 (BR)

Mastrangelo R 460

Mastrangelo S 460

Masuda K 824

Masuda S 836

Masunaga H 1681

Masure M 1616

Mathoulin S 78 (Suppl 2)

Matikainen M 1344

Matsuda K 94, 1052

Matsuda S 768

Matsuda Y 886

Matsumoto K 864

Matsumoto Y 768

Matsumura Y 1235

Matsunobu T 768

Matsuo T 836

Matsuoka H 1317

Matsuura N 1377

Matsuzawa Y 886

Mattes RH 1330

Matthews CE 994

Maula S 244

Maxwell-Armstrong CA 1443

Mayer RJ 441 (L)

Mayne ST 1472

Mayumi T 1564

Mazurenko N 791

Mazzanti R 878

Meagher A 232

Medi F 878

Meert AP 1150

Meijer CJLM 796

Meijer L 283

Mellon JK 270

Melloni E 946

Meng XL 1544

Mercadante S 587

Mercatelli A 878

Mercer AJ 11 (Suppl 1)

Merrouche Y 65 (Suppl 2)

Messori A 303

Meta-Analysis Group in

Cancer 611

Metastron Users Group 297

Metcalfe JC 832

Meynadier J 587

Meza LA 24 (Suppl 1)

Mezzelani A 1087

Michael BD 674

Michaelis J 697

Middleton M 1286 (BR)

Middleton MR 1141

Milano G 579

Mir LM 1272

Mitsudomi T 1199

Mittal KR 975

Mittra I 1286 (BR)

Miura S 1258

Miyahara R 263

Miyake H 859

Miyoshi E 1377

Mizumoto K 864
Mizunuma H 545

M-Kähäri V 659

Mohr P 1036

Mohsin SK 493

Mok SC 352

Mokhtar N 1182

Moll I 819

Møller H 435

Monden M 1377

Monges G 65 (Suppl 2), 74 (Suppl 2)

Monnier A 1179

Mononen N 1344

Monsaert C 1122

Monson JRT 1443

Monsonego J 360

Moore MJ 367

Moore PS 253

Moorghen M 520

Moots P 157

Morales J 1219

Morat L 504

Moreau L 903

Moretti A 636

Moretti R 878

Moretti S 604

Morgan J 80

Morgan Jr RJ 1591

Morgan WF 489

Mori K 1564

Mori M 276

Mori T 1235

Morilla R 1339

Morland B 11, 1029

Morley S 512

Morris TM 1664

Mosca F 1535

Moteleb E 512

Mottot C 360

Mousses S 1635

Muci D 1166

Mueller SC 1330

Mueller W 199

Muers MF 19

Muir G 1512

Muir KM 413

Mukherjee P 1107

Mulder NH 1348

Murai H 1258

Murakami A 851

Murdock M 24 (Suppl 1)

Murphy HS 1076

Murphy LO 926

Murphy MFG 1460

Murphy RF 926

Murray P 1227

Musha T 836

Musk AW 52

Mutanen P 990, 1466

Mutgi AB 1188

Muzzammil T 367

Nagao M 836

Nagase T 886

Nagasue N 1640

Nakagawa S 915, 1564

Nakajima S 1681

Nakamura T 864

Nakamura Y 824, 836

Nakanishi M 824
Nakatani F 768

Nam DK 186

Napp V 19

Narita T 1258

Nashan D 1036

Natale M 1166

Nawata S 851

Nazeyrollas P 1616

Neal DE 270

Negri E 722

Négrier S 81 (Suppl 2)

Nehmé A 1571

Neri B 878

Neuzil J 87

Nevanlinna H 116, 704

New P 157

Newell DR 11, 106, 1289

Ng E-H 1185

Ng EKW 335

Ng Y-P 335

Nicolella G 1166

Niedner H 1571

Nielsen OS 1070

Niklasson A 1193

Nistér M 791

Nobbenhuis MAE 796

Nocera M 808

Noda K 1377

Noda S 886

Noller K 126

Noma H 754

Nooij MA 1577

Nordsmark M 1070

Noss A 571

Nukaya I 94

Numa F 851

Nuzzo V 1586

Nyrén O 120, 965

O’Byrne J 17 (Suppl 1)

O'Connell P 493

O'Day SJ 157

Odze RD 748

Oesterreich S 493

Ogawa M 1497

Ogawa N 443

Ohmori K 1610

Ohtsuka M 920

Oishi T 1551

Okada N 1564

Okamoto T 1235

Okayasu I 209

Okuyama T 1640

Oleinick NL 1099

Oliver RT 340

Ollivier JM 61 (Suppl 2)

On On Chan A 1182

Orbetegli O 196

Orita K 443

Orlandini S 253

Orlowski S 1272

Ørntoft T 1084

Osborne CK 493

Oshima H 1242

Otake Y 263

Ötvös F 926

Ouafik LH 783

Ouyang N 1265

Overgaard J 1070

Overpelt IME 1577
Owens PH 1472

Oyama N 920

Ozcelik H 1635

Paesmans M 1150

Pages S 482

Pairon JC 49 (Suppl 2)

Paju A 1363

Pallaska A 1397

Palmari J 783

Palmer J 126

Palmeri S 1166

Pang T 791

Pani G 529

Panza N 1166

Papworth R 776

Paradiso A 651

Paradiso L 308

Paraschou P 1602

Paraskeva C 520

Parkin D 170

Parkin DM 1207

Parkins CS 565

Parkinson EK 1630

Parnaud G 90

Parry A 1029

Parry J 1308

Paskins L 1512

Patel A 1512

Patricot LM 78 (Suppl 2)

Paul D 813

Paul EM 42

Paul J 170

Paysant J 802

Peach D 1172

Peake MD 19

Pearson ADJ 11, 1029

Pecherstorfer M 344

Pedley RB 1671

Pegelow K 1330

Peiffer G 90

Pein F 604

Pennati M 1387

Perabo FGE 1330

Perez K 714

Pergolizzi R 1528

Perie S 504

Permert J 926

Perrone F 38

Perucho M 743

Pessi MA 1023

Pession A 460

Peter RU 72

Peterse JL 539

Philip T 4 (Suppl 2),

55 (Suppl 2), 78 (Suppl 2)

Phuphanich S 157

Piantedosi F 1166

Piao Z 743

Piccinno R 643

Pierotti MA 1087

Pignatelli M 1656

Pigneux J 31 (Suppl 2)

Pilotti S 539, 1087

Pincheira R 1520

Pinedo HM 550

Pinkerton CR 11

Pinsolle J 37 (Suppl 2)

Pithavala Y 308

Pitsiladis M 11, 308 
Platt FM 1107

Pontén J 791

Portwood N 329

Poulain P 587

Poulter KM 19

Povýšíl C 374

Prete A 460

Price E 1029

Price L 11

Prise KM 674

Prow D 17 (Suppl 1)

Puisieux A 193

Pujol J-L 903

Purdie D 352

Putaud I 1616

Pyrhönen S 897

Qi H 1556

Quantin X 903

Quereux C 1616

Quinlan RA 512

Quoix E 903

Radbruch L 587

Radford JA 465

Raju U 975

Ramirez AJ 1172

Ranes MK 1107

Ranson M 465

Rapakko K 116

Rarick M 24 (Suppl 1)

Raschko JW 1591

Ratashak A 1412

Ratcliffe D 1011

Rath GK 1016

Rath P 475

Raoul JL 74 (Suppl 2)

Ravaioli A 1023

Ray I 18 (Suppl 2)

Raymond E 631

Reardon D 1591

Recaldin E 1023

Recht L 157

Reed MWR 1384

Reed NS 170

Regnard JF 51 (Suppl 2)

Reif S 1453

Remiddi F 529

Remontet L 1477

Renault-Salis JL 37 (Suppl 2)

Resbeut M 24 (Suppl 2), 51 (Suppl 2)

Reymond MO 783

Ria F 529

Ricci S 636

Richards D 17 (Suppl 1)

Richards MA 1172

Ricker W 126

Rigacci L 303

Rigas J 17 (Suppl 1)

Rigaud G 253

Riordan HD 1544

Riordan NH 1544

Ripamonti C 587

Risse EKJ 796

Ristamäki R 244

Ritchie LD 910

Riva C 1087
Rivière A 1179

Rivoire M 65 (Suppl 2)

Robanus-Maandag EC 539

Robbins A 465

Roberts SA 776, 892

Robins RA 1443

Robinson BWS 52

Robinson D 435

Roca i Casas J 587

Rodier JF 31 (Suppl 2)

Rodriguez AC 1219

Rogers L 157

Rohatiner AZS 147 (L)

Romain S 783

Romano S 878

Roncalli M 196

Ronen SM 691

Rose C 783

Rosing H 42

Ross G 1043

Rosselli R 529

Rossi A 38

Rostagno P 579

Rostami-H A 1126

Rougier P 61 (Suppl 2), 65

(Suppl 2), 74 (Suppl 2)

Roukos DH 1602

Rouquet C 1677

Rouse A 1308

Roussel A 61 (Suppl 2)

Rowan A 1314

Roy HK 1412

Rozendaal L 796

Ruan Z-X 994

Rubinstein LV 1424

Rudenstam C-M 1156

Rufini V 460

Ruffié P 49 (Suppl 2),

51 (Suppl 2)

Ruggieri-Pignon S 65 (Suppl 2)

Ruiters MHJ 1348

Rumi MAK 1640

Rumpold G 179

Rush R 594

Russell C 157

Russo A 1166

Rustin G 1043

Ryan DP 441 (L)

Rybczynska M 1405

Ryberg D 226

Saarialho-Kere U 659

Saaristo R 164

Saarto T 1047

Sabatier L 504

Saegusa M 209

Saga T 1681

Saito M 736 (L)

Saito T 1564

Saito Y 1235

Sakahara H 1681

Sakakura C 824

Sakamoto A 1317

Sakata I 1681

Sakon M 1377

Salo T 1363

Salvetti A 636

Sambrook RJ 1447

Sandstedt B 1505

Sanghvi V 739
Saranath D 739

Sargent JM 680, 959

Sarradat A 61 (Suppl 2)

Sasaki R 836

Sasatomi T 915

Sastre-Garaud X 24 (Suppl 2)

Sato H 1640

Sato $S 1551$

Satoh M 920

Saul J 48, 1011

Saurel J 360

Sausville EA 1424

Saven A 17 (Suppl 1), 24 (Suppl 1)

Sawe J 587

Scarpa A 253

Scerrati M 529

Schally AV 926

Schätzle S 283

Scheeren JW 550

Schellens JHM 42

Schepartz S 1424

Schiffman M 1219

Schleutker J 1344

Schlumberger M 808

Schmidt D 1330

Schmidt T 1076

Schmidt TL 1544

Schmitz U 199

Scholefield JH 1443

Schriber J 1591

Schröder CP 1348

Schuller DE 237

Schüller J 1043

Schuller J 11 (Suppl 1)

Schulz TF 122

Schumacher U 819

Schüz J 697

Schwartz JL 489

Schwartzberg L 17 (Suppl 1)

Scorilas A 643, 760, 1488

Scott D 776, 892

Scott LJ 1417

Scottish Gynaecological Cancer

Trials Group 170

Scottish Melanoma Group 1146

Sculier JP 1150

Sebastian G 1036

Sebban C 55 (Suppl 2)

Seddighzadeh M 329

Seenu V 1016

Seibel MJ 344

Seifeldin IA 1182

Seigneurin JM 783

Seiter S 1036

Seitz JF 61 (Suppl 2)

Selby P 1293

Sellakumar G 1571

Senoo M 1235

Seong C-M 1252

Sersa G 565

Sette A 94

Sévenet N 199

Seyfried TN 1107

Shamash J 340

Shanks JH 1417

Shapiro W 157

Sharma SK 1671

Sharom FJ 1405

Sharp L 910
Sheedy B 308

Sherman ME 1219

Sheu JC 743

Shibahara T 754

Shibata SI 1591

Shichijo S 915

Shimada M 1551

Shimada S 1497

Shimomura K 824

Shin BA 844

Shiomori K 1497

Shipman CM 1126

Shoker BS 1064

Shore RE 975

Shu X-O 994

Sibson DR 1064

Signorello LB 965

Silcocks P 728, 1215

Siliani L 878

Silvy M 936

Simon D 743

Simone G 651

Símová J 374

Singh Jadeja J 17 (Suppl 1)

Sivagnanasundaram S 218

Skaug V 226

Skliris GP 1095

Slevin N 776

Slevin NJ 8

Sloane JP 1064

Šmahel M 374

Smith GA 832

Smith K 1322

Smith P 1293

Smith RE Jr 24 (Suppl 1)

Smyrk TC 1412

Smyth JF 1146

Snaddon J 1630

Snijders PJF 1058

Sobotková E 374

Sobrero A 1023

Sölétormos G 1301

Soliman AS 1182

Söllner W 179

Somlo G 1591

Sone $\mathrm{S} 25$

Song E 1265

Sorahan T 141

Soria C 802

Soria JC 504

Soria J-C 631

Sorsa T 1363

Soukop M 465

Soussi T 57

Sozzi G 1087

Speirs V 1095

Spendlove I 80

Spitaler M 1405

Sprinzl G 179

Spry N 297

Spyratos F 783

Srivastava A 1016

Stanson J 1624

Staratschek-Jox 381

Steel CM 594

Steels E 1150

Steineck G 329, 1505

Steiner G 1330

Steiner RA 33

Steinfels E 1405 
Steixner E 179

Stel AJ 1115

Stenman U-H 1363

Stevens A 1308

Stevenson S 52

Stewart M 600

Stiggelbout AM 1577

Stiller CA 141, 406

Stines J 55 (Suppl 2)

Stolte H 313

Stolz W 1036

Stoppa-Lyonnet D 482

Storme G 6 (Suppl 2)

Storme GA 1122

Suefuji Y 915

Sugimachi K 276

Sugiura T 1199

Sugiyama M 1317

Sulkes J 478

Sullivan M 1156

Suminami Y 851

Sun XS 1179

Sung JJY 335

Sweetenham J 1291

Swerdlow AJ 406

Swinnen L 157

Synold T 1591

Syrjäkoski K 116, 1344

Szekely L 122

Taché S 90

Tada A 1564

Tada T 736 (L)

Taft C 149

Taguchi O 1258

Tajima K 1199

Takagi K 1242

Takahashi K 736 (L)

Takahashi M 1551

Takama F 545

Takashima S 25

Takeda H 1681

Takeda T 1377

Takehara Y 1681

Takesako K 94

Takeuchi T 1258

Takezaki T 1199

Talbot I 218

Talieri M 1488

Tamborini E 1087

Tammela TLJ 1344

Tamminen A 704

Tamura S 886

Tamura Y 1564

Tanaka F 263

Tanaka H 94, 836, 1052

Tanaka K 768

Tanaka M 864

Tanaka N 443

Tandle AT 739

Tanimura H 94, 1052

Tannapfel A 982

Tannock IF 100

Tanzawa H 754

Tarquini R 878

Tashima S 1497

Taylor CG 680, 959

Taylor DD 1624

Taylor GA 11

Tchekmedyian NS 24 (Suppl 1)
Tedeschi R 122

Ten Bokkel Huinink WW 42

Teppo L 122

Terakawa N 1551

Ternier F 24 (Suppl 2)

Terry P 120

Tervahartiala T 1363

Testa NG 1417

Testore P 1023

Tetef ML 1591

Thakore KS 748

Thatcher N 1141

Theobald S 8 (Suppl 2)

Theodor L 475

Thibout D 802

Thies A 819

Thiesse P 78 (Suppl 2)

Thomas G 748

Thomas GA 832

Thomas L 18 (Suppl 2), 31 (Suppl 2)

Thomas RK 381

Thornalley PJ 670

Tiitinen A 897

Tilgen W 1036

Tisdale MJ 1135, 1648

Titus-Ernstoff L 126, 714

To K-F 335

Toledano MB 1482

Tominaga O 57

Tomlinson I 232

Tomlinson IPM 1314

Toniolo P 975

Tornesello A 460

Torres-Duarte A 1599

Tosi M 482

Tounekti O 1272

Tourne ME 1391

Tournemaine N 18 (Suppl 2)

Tozer GM 565

Tranga T 1488

Tretli S 1463

Trippoli S 303

Troncone L 460

Tsai JF 709

Tsuchiya I 1235

Tsujimoto M 1377

Tsujino M 1564

Tsunoda T 94, 1052

Tsuruta J 1497

Turci D 1023

Turner SL 297

Tut VM 270

Tuxen MK 1301

Twycross RG 587

Tynes T 397

Udart M 72

Ugurel S 1036

Ulrich J 1036

Umano Y 94, 1052

Underwood M 1512

Unsal K 57

Utikal J 72

Utsumi H 836

Uzawa K 754

Vähäkangas K 116

Vahteristo P 116, 704

Vaiani M 303
Vaittinen P 388

Välimäki M 1047

Valle JW 875

Vallejo C 651

Vallot F 1150

van de Velde CJH 64

van de Vijver MJ 539

van den Bent MJ 157

Van den Berge DL 1122

van den Brule AJC 796

van der Graaf WTA 1348

van der Meulen-Muileman IH 550

van der Zee AGJ 1348

van Duin M 1058

van Krieken JHJM 64

van Lohuizen M 1372

van Slooten H 1577

Van Tellingen O 604

Varadarajan P 1571

Varani J 1076

Varghese C 1215

Varley JM 321

Vasey PA 170

Vassal G 604

Vasse M 802

Vatten LJ 417

Vaughan Hudson G 1293

Veal GJ 1029

Vehmanen L 1047

Veltri E 38

Venables RS 512

Venditti JM 1424

Ventafridda V 587

Verheijen RHM 796

Verovski VN 1122

Vian L 1677

Villa E 881

Vilmer C 81 (Suppl 2)

Vincent P 31 (Suppl 2)

Vincent TJ 1460

Vitale G 1586

von Deimling A 199

von Ruecker A 1330

von Vangerow A 313

Vonka V 374

Vonlanthen S 1372

Voog E 18 (Suppl 2)

Voorhorst FJ 796

Wacholder S 1219

Wada H 263

Wahlström T 897, 1363

Wainer IW 1599

Wakefield J 1482

Walch A 202

Walker M 1512

Wall L 600

Wallgren A 1193

Walt H 33

Walters JRF 218

Wang D 545

Wang H 748

Wang LY 709

Wang M 1265

Wang Q 193

Ward RL 232

Warnes SL 571

Warren C 892

Wasan H 1314
Watanabe T 1182

Waters J 1

Watson SA 1664

Weber C 87

Weber M 199

Weber T 87

Webley SD 1671

Weihrauch M 982

Welbank H 1029

Wellmann J 134

Werner M 202

West CM 892

Whiteman D 1460

Whiteside TL 1624

Whitley E 728

Whittle H 1207

Wichmann HE 134

Wijkström H 329, 1505

Wiklund TA 244

Wikman FP 1084

Williams AC 520

Williams ED 832

Williamson CJ 680, 959

Winqvist R 116

Winter H 1215

Wirger A 1330

Wisman GBA 1348

Withoff S 1115

Wittekind C 982

Woitge HW 344

Wojno KJ 1076

Wolf J 381

Wolk A 120

Wong E 157

Wong H 493

Woodford-Richens KL 1314

Wrbitzky R 982

Wright JG 452

Wunder JS 1635

Wyld L 1384

Xu D 621

Xu K 670

Xu Z-G 920

Yakushiji T 754

Yamada T 263

Yamagishi H 824

Yamamoto A 1564

Yamamoto N 754

Yamanda T 25

Yamasaki E 886

Yamashita 276

Yamaue H 94, 1052

Yanagihara K 263

Yang Z-G 25

Yardley C 959

Yasuda T 1610

Yasuda Y 836

Yasuoka R 824

Yatabe Y 1199

Yen Y 1591

Yeremin L 475

Yi JW 186

Yi Q 621

Ylikorkala O 897

Yoshida K-I 1242

Yoshida T 209

Yoshimoto M $736(\mathrm{~L})$

Yoshimura T 400 
Young T 1172

Yousef GM 643

Yudoh K 1610

Yule SM 1029

Yung WKA 1252

Zaffaroni N 1387

Zaharevitz D 1424
Zahm SH 1472

Ž́k R 374

Zalcman G 57

Zamboni G 253

Zander T 381

Zankl H 283

Zeleniuch-Jacquotte A 975

Zenita K 1258
Zerat L 360

Zhang B 1472

Zhang J-T 1520

Zhang Q 493, 1571

Zhang Y 1472

Zheng C 621

Zheng T 1472

Zheng W 994
Zhou H 951

Ziegler R 344

Zienolddiny S 226

Zitzelsberger H 202 